\section{RR runter, Harnsäure rauf Zipperlein vom Blutdrucksenker}

Eine Hypertonie geht per se mit einem erhöhten Gichtrisiko einher. Zusätzlich steigern fast alle Antihypertensiva das Erkrankungsrisiko. Davon ausgenommen sind nur die Kalziumkanalblocker und der AT1-Antagonist Losartan: Sie verfügen sogar über eine präventive Wirkung.

Die Auswirkungen einer blutdrucksenkenden Therapie auf das Gichtrisiko wurden in einer Fall-Kontroll-Studie mit Daten von britischen Allgemeinarztpraxen untersucht.

24.768 Patienten mit neu diagnostizierter Gicht wurde eine Stichprobe von passenden Kontrollpersonen gegenübergestellt. Bei gut der Hälfte der Gicht- kranken bestand auch ein Bluthochdruck. Hypertoniker hatten, nach dem Abgleich anderer Risikofaktoren, ein um 75\% höheres Gichtrisiko als Personen mit normalem Blutdruck.

\section{Kalziumantagonisten schützen}

Hochdruckpatienten, die einen Kalziumkanalblocker oder Losartan erhielten, entwickelten seltener eine Gicht. Ihr Risiko war um 13\% bzw. 19\% niedriger als das von Hochdruckpatienten ohne diese Medikamente. Das entspricht in absoluten Zahlen einem Rückgang um 60 bzw. 86 Fälle pro 100.000 Personenjahren. Eine vergleichbare Schutzwirkung zeigte die Einnahme von Kalziumantagonisten und Losartan bei
Patienten mit einer anderen Indikation als einer Hypertonie. Generell fiel der Effekt umso deutlicher aus, je länger die Behandlung dauerte und je höher sie dosiert war.

\section{Losartan setzt am Tubulus an}

Die präventive Wirkung von Kalziumantagonisten lässt sich wahrscheinlich durch den Anstieg der glomerulären Filtrationsrate und die vermehrte Ausscheidung von Harnsäure erklären. Der harnsäuresenkende Effekt von Losartan wurde schon in anderen Untersuchungen als vergleichbar mit klassischen Urikosurika wie Probenecid beschrieben und setzt vermutlich am Harnsäuretransport in den Tubuluszellen an.

Andere AT1-Antagonisten und ACEHemmer verfügen nicht über diesen Wirkmechanismus. Diuretika erhöhen die Rückresorption von Harnsäure am proximalen Tubulus und können bereits innerhalb weniger Tage zu einem Anstieg der Harnsäurewerte führen. Warum Betablocker den Harnsäurespiegel steigen lassen, ist nicht bekannt. (bs) BMJ 2011, 344:d8190

\title{
Gefährlicher Aufschnitt Wurst und Speck locken Pankreaskrebs
}

\begin{abstract}
Wenn es an der Wursttheke immer gern ein bisschen mehr sein darf, kann der Einkauf teuer werden. Denn mit der Menge des Aufschnitts wächst das Risiko, an Krebs der Bauchspeicheldrüse zu erkranken - und der kostet die Betroffenen meist das Leben.
\end{abstract}

Wer sich täglich eine 50-Gramm-Portion von Erzeugnissen aus verarbeitetem Fleisch - wie Wurst, Schinken oder Speck - zusätzlich auf den Teller legt, erhöht damit die Gefahr, ein Pankreaskarzinom zu entwickeln um $19 \%$, so eine
Metaanalyse einschlägiger prospektiver Studien. Auch rotes Fleisch ist riskant das gilt allerdings nur für Männer: Eine Zunahme des Verzehrs um $120 \mathrm{~g}$ pro Tag lässt die Gefahr um rund 30\% steigen. Die Follow-up-Zeiträume der untersuchten Studien lagen dabei zwischen fünf und 20 Jahren.

\section{Vierthäufigste \\ Krebstodesursache}

Freilich sind die Zahlen vor dem Hintergrund der Erkrankungsrate zu sehen. Sie liegt für Krebs des Pankreas in
Deutschland laut den aktuellen Zahlen des Robert Koch-Instituts und der deutschen Krebsregister, die sich auf 2006 beziehen, bei 15,8/100.000 Männer bzw. bei 16,6/100.000 Frauen (jeweils rohe Rate). Andererseits ist zu beachten, dass die Heilungsaussichten denkbar gering sind und die Mortalitätsziffer der Inzidenz praktisch gleichkommt. Das macht den Pankreaskrebs zur vierthäufigsten Krebstodesursache in Deutschland, obwohl er auf der Liste der Neuerkrankungen nur Rang neun (Frauen) bzw. zehn (Männer) belegt. Br J Cancer 2012 (online first) (rb) 